\title{
Numerical-analytical calculation of a cylindrical reservoir taking into account creep
}

\author{
Anton Chepurnenko*, Tatyana Polyakova, Marina Rozen, and Marina Grigoryan
}

Don State Technical University, 344002, Rostov-on-Don, Russia

\begin{abstract}
The article proposes a numerical-analytical solution to the problem of axisymmetric loading of the closed cylindrical shell, taking into account the creep of the material. The calculation is performed using the functions of A.N. Krylov in combination with the method of Euler and Runge-Kutta of the fourth order. Comparison with the solution using the finite difference method is presented.
\end{abstract}

\section{Introduction}

In paper [1] we earlier considered the problem of calculating the axis symmetrically loaded circular cylindrical shell (figure 1) taking into account the creep of the material. The problem was reduced to a fourth-order differential equation with respect to deflection $w$ :

$$
D \frac{d^{4} w}{d x^{4}}+\frac{E h w}{R^{2}}=q-\frac{d^{2} M_{x}^{*}}{d x^{2}}+\frac{1}{R}\left(N_{\theta}^{*}-v N_{x}^{*}\right)
$$

where $D=\frac{E h^{3}}{12\left(1-v^{2}\right)}-$ cylindrical stiffness, $E$ and $v$ - respectively, the modulus of elasticity and the Poisson's ratio of the material, $h$ - shell thickness,

$$
N_{x}^{*}=\frac{E}{1-v^{2}} \int_{-h / 2}^{h / 2}\left(\varepsilon_{x}^{*}+v \varepsilon_{\theta}^{*}\right) d z ; N_{\theta}^{*}=\frac{E}{1-v^{2}} \int_{-h / 2}^{h / 2}\left(\varepsilon_{\theta}^{*}+v \varepsilon_{x}^{*}\right) d z, \quad M_{x}^{*}=\frac{E}{1-v^{2}} \int_{-h / 2}^{h / 2}\left(\varepsilon_{x}^{*}+v \varepsilon_{\theta}^{*}\right) z d z
$$

Equation (1) was solved numerically by the finite difference method. However, in this problem, there is a pronounced edge effect at the base of the shell, and therefore a sufficiently dense mesh is required for the correct calculation.

In this article, we propose the numerical-analytical method that allows one to more accurately take into account the edge effect in the support zone.

\footnotetext{
*Corresponding author: anton_chepurnenk@mail.ru
} 


\section{Methods}

Let us introduce the dimensionless coordinate $\xi$ determined by the formula:

$$
\xi=\alpha x
$$

where $\alpha$ is a coefficient determined by the formula:

$$
\alpha=\sqrt[4]{\frac{E h}{4 D R^{2}}}
$$
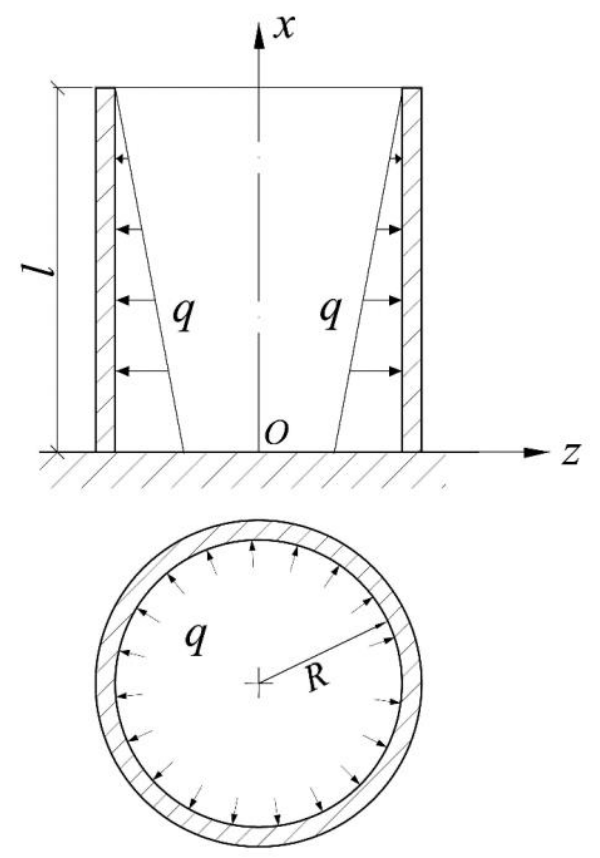

Fig. 1. Calculation scheme

The transition from derivatives with respect to $x$ to derivatives with respect to $\xi$ is carried out as follows:

$$
\begin{gathered}
\frac{d f}{d x}=\frac{d f}{d \xi} \frac{d \xi}{d x}=\alpha \frac{d f}{d \xi} ; \\
\frac{d^{n} f}{d x^{n}}=\alpha^{n} \frac{d^{n} f}{d \xi^{n}} .
\end{gathered}
$$

Equation (1) can be rewritten as:

$$
\frac{d^{4} w}{d \xi^{4}}+4 w=\frac{1}{\alpha^{4} D}\left(q-\alpha^{2} \frac{d^{2} M^{*}}{d \xi^{2}}+\frac{1}{R}\left(N_{\theta}^{*}-v N_{x}^{*}\right)\right)
$$

The solution to equation (5) can be represented as: 


$$
w=C_{1} Y_{1}+C_{2} Y_{2}+C_{3} Y_{3}+C_{4} Y_{4}+\bar{w}_{0}+\bar{w}^{*},
$$

where $Y_{1}, Y_{2}, Y_{3}, Y_{4}$ are the functions of academician A.N. Krylov:

$$
\begin{aligned}
& Y_{1}(\xi)=\cosh \xi \cos \xi \\
& Y_{2}(\xi)=\frac{1}{2}(\cosh \xi \sin \xi+\sinh \xi \cos \xi) \\
& Y_{3}(\xi)=\frac{1}{2} \sinh \xi \sin \xi \\
& Y_{4}=\frac{1}{4}(\cosh \xi \sin \xi-\sinh \xi \cos \xi),
\end{aligned}
$$

$\bar{w}_{0}$ - the particular solution of equation (5) without taking into account creep, $\bar{w}^{*}-$ an additive to a particular solution related to creep.

The function $\bar{w}_{0}$ can be expressed through the functions of A.N. Krylov by integral:

$$
\bar{w}_{0}=\frac{1}{\alpha^{4} D} \int_{0}^{\xi} Y_{4}(\xi-t) q(t) d t
$$

For a triangular diagram of water pressure, the load function in dimensionless coordinates is written as:

$$
q(\xi)=\frac{\gamma}{\alpha}(\lambda-\xi)
$$

After substituting (9) into (8), the particular solution $\bar{w}_{0}$ takes the form:

$$
\bar{w}_{0}=\frac{\gamma}{4 \alpha^{5} D}\left(\lambda-\xi-\lambda Y_{1}+Y_{2}\right)
$$

The function $\bar{w}^{*}$ is defined as follows:

$$
\bar{w}^{*}=\frac{1}{\alpha^{4} D} \int_{0}^{\xi}\left(-\left.\alpha^{2} \frac{d^{2} M^{*}}{d \xi^{2}}\right|_{\xi=t}+\frac{1}{R}\left(N_{\theta}^{*}(t)-v N_{x}^{*}(t)\right)\right) Y_{4}(\xi-t) d t .
$$

To integrate expression (11), a uniform mesh with respect to $x$ is introduced. Integration is performed numerically using the trapezium or Simpson method. The angle of rotation is written as:

$$
\varphi=\frac{d w}{d x}=\alpha\left(-4 C_{1} Y_{4}+C_{2} Y_{1}+C_{3} Y_{2}+C_{4} Y_{3}+\frac{\gamma}{4 \alpha^{5} D}\left(-1+4 \lambda Y_{4}+Y_{1}\right)\right)+\frac{d \bar{w}^{*}}{d x}
$$

The bending moment is determined as follows: 


$$
\begin{gathered}
M_{x}=-D \frac{d^{2} w}{d x^{2}}-M_{x}^{*}=-\alpha^{2} D\left(-4 C_{1} Y_{3}-4 C_{2} Y_{4}+C_{3} Y_{1}+C_{4} Y_{2}+\frac{\gamma}{\alpha^{5} D}\left(\lambda Y_{3}-Y_{4}\right)\right)- \\
-D \frac{d^{2} \bar{w}^{*}}{d x^{2}}-M_{x}^{*} . \\
Q_{x}=\frac{d M_{x}}{d x}=-\alpha^{3} D\left(-4 C_{1} Y_{2}-4 C_{2} Y_{3}-4 C_{3} Y_{4}+C_{4} Y_{1}+\right. \\
\left.+\frac{\gamma}{\alpha^{5} D}\left(\lambda Y_{2}-Y_{3}\right)\right)-D \frac{d^{3} \bar{w}^{*}}{d x^{3}}-\frac{d M_{x}^{*}}{d x} .
\end{gathered}
$$

The boundary conditions for the shell rigidly clamped at the base are:

$$
\begin{aligned}
& \text { at } x=0: w=0, \frac{d w}{d x}=0 \\
& \text { at } x=h: M_{x}=-D \frac{d^{2} w}{d x^{2}}-M_{x}^{*}=0, Q_{x}=\frac{d M_{x}}{d x}=-D \frac{d^{3} w}{d x^{3}}-\frac{d M_{x}^{*}}{d x}=0 .
\end{aligned}
$$

Substituting the boundary conditions (15) into expressions (6), (12) - (14), we obtain:

$$
\begin{aligned}
& w(0)=0 \rightarrow C_{1}=-\bar{w}^{*}(0)=0 \\
& \varphi(0)=0: \alpha C_{2}+\left.\frac{d \bar{w}^{*}}{d x}\right|_{x=0}=0 \rightarrow C_{2}=-\left.\frac{1}{\alpha} \frac{d \bar{w}^{*}}{d x}\right|_{x=0}=-\left.\frac{d \bar{w}^{*}}{d \xi}\right|_{\xi=0} . \\
& M_{x}(\lambda)=0:-\left.M_{x}^{*}\right|_{x=l}-\alpha^{2} D\left(\left.\frac{4}{\alpha} \frac{d \bar{w}^{*}}{d x}\right|_{x=0} Y_{4}(\lambda)+C_{3} Y_{1}(\lambda)+C_{4} Y_{2}(\lambda)+\right. \\
& \left.+\frac{\gamma}{\alpha^{5} D}\left(\lambda Y_{3}(\lambda)-Y_{4}(\lambda)\right)\right)-\left.D \frac{d^{2} \bar{w}^{*}}{d x^{2}}\right|_{x=l}=0 ; \\
& Q_{x}(\lambda)=0:=-\alpha^{3} D\left(\left.\frac{4}{\alpha} \frac{d \bar{w}^{*}}{d x}\right|_{x=0} Y_{3}(\lambda)-4 C_{3} Y_{4}(\lambda)+C_{4} Y_{1}(\lambda)+\right. \\
& \left.+\frac{\gamma}{\alpha^{5} D}\left(\lambda Y_{2}(\lambda)-Y_{3}(\lambda)\right)\right)-\left.D \frac{d^{3} \bar{w}^{*}}{d x^{3}}\right|_{x=l}-\left.\frac{d M_{x}^{*}}{d x}\right|_{x=l}=0 .
\end{aligned}
$$

Thus, at each time step, we have two equations with two unknowns to determine the constants $C_{3}$ and $C_{4}$.

The creep deformations at each step are determined from the deformations and stresses at the previous step using the fourth-order Runge-Kutta method or Euler method. The procedure for determining creep deformations is described in more detail in [2-9].

\section{Results and Discussion}

The polymer shell from recycled PVC was calculated with the same initial data as in [1] $: h$ $=1 \mathrm{~cm}, l=3 \mathrm{~m}, R=2 \mathrm{~m}, \gamma=10 \mathrm{kN} / \mathrm{m}^{3}, E=1480 \mathrm{MPa}, v=0.3$. The nonlinear MaxwellGurevich equation was used as the creep law, which in cylindrical coordinates, taking into account axial symmetry, is written in the form: 


$$
\frac{\partial \varepsilon_{i}^{*}}{\partial t}=\frac{f_{i}^{*}}{\eta^{*}}, i=x, \theta
$$

where $f_{i}^{*}$ is the stress function, $\eta^{*}$ is the relaxation viscosity.

$$
f_{i}^{*}=\frac{3}{2}\left(\sigma_{i}-p\right)-E_{\infty} \varepsilon_{i}^{*}
$$

where $p=\left(\sigma_{\mathrm{x}}+\sigma_{\theta}\right) / 3$ is the average stress, $E_{\infty}$ is the high elasticity modulus.

$$
\frac{1}{\eta^{*}}=\frac{1}{\eta_{0}^{*}} \exp \left(\frac{\left|f^{*}\right|_{\max }}{m^{*}}\right)
$$

where $\eta_{0}^{*}$ is the initial relaxation viscosity, $m^{*}$ is the velocity modulus.

Rheological parameters of $\mathrm{PVC}$ at the temperature of $20^{\circ} \mathrm{C}$ : high elasticity modulus $E_{\infty}$ $=5990 \mathrm{MPa}$, velocity modulus $m^{*}=12.6 \mathrm{MPa}$, initial relaxation viscosity $\eta_{0}^{*}=9.06 \cdot 10^{5}$ $\mathrm{MPa} \cdot \min$ - were determined previously in paper [10].

Figure 2 shows the graphs of the time variation of the maximum deflection value (at the point $x=0.32 \mathrm{~m}$ ), obtained using the Euler method for a different number of time steps $n$. This figure also shows a curve calculated on the basis of the Runge-Kutta method when the time interval is divided into 5 segments. The Runge-Kutta method has a significantly faster convergence, in the considered problem, 16 times fewer steps were required to obtain an acceptable result, however, when using it, at each step, it is required to determine the stresses 4 times.

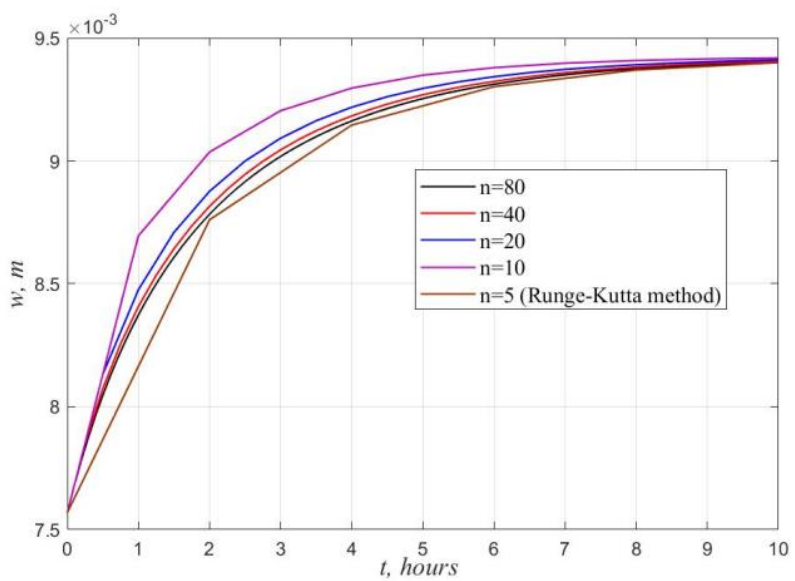

Fig. 2. Graphs of changes in the maximum deflection for a different number of steps $n$ in time

Comparison of the results obtained using finite differences method (FDM) and numerical-analytical calculation is presented in Table 1.

Table 1. Comparison of the largest displacements at different points in time, obtained using the finite differences method $\left(w_{1}\right)$ and the numerical-analytical approach $\left(w_{2}\right)$

\begin{tabular}{|c|c|c|c|c|c|c|c|c|}
\hline$t$, hours & 1 & 2 & 3 & 4 & 5 & 6 & 7 & 8 \\
\hline $\mathrm{w}_{1}, \mathrm{~m}$ & 8.3732 & 8.7825 & 9.0182 & 9.1622 & 9.2532 & 9.3118 & 9.3500 & 9.3751 \\
\hline $\mathrm{w}_{2}, \mathrm{~m}$ & 8.3839 & 8.7946 & 9.0312 & 9.1758 & 9.2671 & 9.3260 & 9.3644 & 9.3896 \\
\hline
\end{tabular}


The largest discrepancy between the results is $0.15 \%$, which indicates their reliability.

\section{Conclusion}

A numerical-analytical solution to the problem of calculating the wall of a cylindrical reservoir under axisymmetric loading taking into account creep is proposed. A test problem for a polymer shell is solved, followed by comparison of the results with the solution using the finite difference method. The discrepancy between the results is insignificant. The proposed approach makes it possible to more accurately calculate the stresses in the support zone.

\section{References}

1. V.I. Andreev, A.S. Chepurnenko, B.M. Yazyev, Procedia Engineering 165, 1141-1146 (2016)

2. V. Chepurnenko, B. Yazyev, X. Song, MATEC Web of Conferences 129, 05009 (2017)

3. S. B. Yazyev et al., MATEC Web of Conferences 129, 05010 (2017)

4. V. Chepurnenko, B. Yazyev, I. Dubovitskaya, E3S Web of Conferences 164, 02003 (2020)

5. S.V. Litvinov et al., International Polymer Science and Technology 42, 23-25 (2015)

6. S. B. Yazyev et al., IOP Conference Series: Materials Science and Engineering 913 (2), 022021 (2020)

7. V.S. Chepurnenko, B.M. Yazyev, International Journal for Computational Civil and Structural Engineering 15, 25-33 (2019).

8. L. Trush et al., Energy Management of Municipal Transportation Facilities and Transport. Springer, Cham, pp. 885-893 (2017)

9. S. Litvinov et al., Energy Management of Municipal Transportation Facilities and Transport. Springer, Cham, pp. 902-907 (2017)

10. A.E. Dudnik, A.S. Chepurnenko, S.V. Litvinov, International Polymer Science and Technology 44, 30-33 (2017) 